\title{
Ordering blood tests for patients with unexplained fatigue in general practice: what does it yield? Results of the VAMPIRE trial
}

Hèlen Koch, Marloes A van Bokhoven, Gerben ter Riet, JM Tineke van Alphen-Jager,

Trudy van der Weijden, Geert-Jan Dinant and Patrick JE Bindels

\begin{abstract}
Background

Unexplained fatigue is frequently encountered in general practice. Because of the low prior probability of underlying somatic pathology, the positive predictive value of abnormal (blood) test results is limited in such patients.

Aim

The study objectives were to investigate the relationship between established diagnoses and the occurrence of abnormal blood test results among patients with unexplained fatigue; to survey the effects of the postponement of test ordering on this relationship; and to explore consultation-related determinants of abnormal test results.

Design of study

Cluster randomised trial.

Setting

General practices of 91 GPs in the Netherlands.

Method

GPs were randomised to immediate or postponed bloodtest ordering. Patients with new unexplained fatigue were included. Limited and expanded sets of blood tests were ordered either immediately or after 4 weeks. Diagnoses during the 1-year follow-up period were extracted from medical records. Two-by-two tables were generated. To establish independent determinants of abnormal test results, a multivariate logistic regression model was used. Results

Data of 325 patients were analysed $(71 \%$ women; mean age 41 years). Eight per cent of patients had a somatic illness that was detectable by blood-test ordering. The number of false-positive test results increased in particular in the expanded test set. Patients rarely reconsulted after 4 weeks. Test postponement did not affect the distribution of patients over the two-by-two tables. No independent consultation-related determinants of abnormal test results were found.

\section{Conclusion}

Results support restricting the number of tests ordered because of the increased risk of false-positive test results from expanded test sets. Although the number of reconsulting patients was small, the data do not refute the advice to postpone blood-test ordering for medical reasons in patients with unexplained fatigue in general practice.

\section{Keywords}

fatigue; diagnostic tests; medically unexplained symptoms; sensitivity and specificity.
\end{abstract}

\section{INTRODUCTION}

In $3-39 \%$ of the patients visiting a GP with complaints, the GP is unable to establish a diagnosis after taking their history and performing a physical examination. Such complaints are labelled 'unexplained complaints' within the definition of the Dutch College of General Practitioners. ${ }^{1}$ 'Unexplained fatigue' is the most common unexplained complaint presented in general practice. $^{2}$ New unexplained complaints are often assumed to be mild and self-limiting. ${ }^{3}$ Sometimes, however, they may be indicative of somatic or psychiatric pathology, or evolve into chronic forms of unexplained complaints, such as somatoform disorders.

It has frequently been suggested that immediate test ordering in unexplained complaints is superfluous. ${ }^{4-6}$ As the prior probability of underlying

H Koch, MD, PhD, post doc; G ter Riet, MD, PhD, associate professor, Academic Medical Center-University of Amsterdam, Division of Clinical Methods and Public Health, Department of General Practice, Amsterdam, the Netherlands. MA van Bokhoven, $M D, P h D$, senior post doc; T van der Weijden, $M D, P h D$, associate professor; GJ Dinant, $M D$, professor of general practice, Maastricht University, School for Primary Care and Public Health (CAPHRI), Department of General Practice, Maastricht, the Netherlands. JM van Alphen-Jager, chemical pathologist, director of Medial, Diagnostic Laboratories, Haarlem, the Netherlands. PJE Bindels, MD, professor of general practice, Erasmus Medical Center, Department of General Practice, Rotterdam, the Netherlands.

Address for correspondence

Hèlen Koch, Academic Medical Center-University of Amsterdam, Division of Clinical Methods and Public Health, Department of General Practice, Amsterdam, the Netherlands. E-mail: h.koch@amc.uva.nl

Submitted: 15 May 2008; Editor's response: 7 July 2008; final acceptance: 24 November 2008.

(c)British Journal of General Practice 2009.

This is the full-length article of an abridged version published in print. Cite this article as: Br J Gen Pract 2009; DOI: 10.3399/bjgp09X420310 
somatic pathology may be expected to be less than $5 \%$ in unselected patients, ${ }^{7}$ the likelihood of falsepositive test results is high. Therefore, in its guideline on blood-test ordering, the Dutch College of General Practitioners recommends a 4-week postponement of blood-test ordering for patients with unexplained complaints. If blood tests are ordered, it recommends ordering a limited number of tests. This approach is expected to lower the number of tested patients and, consequently, the risk of false-positive test results. A potential downside is that the diagnosis of serious illnesses may be delayed or missed altogether. The guideline is consensus based with respect to the recommendations given. Little is known about the actual diagnostic value of immediate or postponed blood-test ordering in patients with unexplained complaints.

The VAgue Medical Problems In REsearch (VAMPIRE) trial was designed to learn more about patients with unexplained complaints in general practice and about the value of blood-test ordering for these patients. The aim of this paper is to describe the course of new unexplained fatigue in general practice, in terms of established diagnoses over time and with regard to the occurrence of abnormal test results in patients with new unexplained fatigue. Furthermore, the study aimed to assess to what extent immediate or postponed blood-test ordering would affect this course. A final aim was to explore which consultation-related determinants, if any, predict abnormal blood test results. Such a model, combined with other evidence of the value of blood-test ordering, might help the GP to choose rationally when ordering blood tests for patients with unexplained fatigue.

\section{METHOD}

The protocol of the VAMPIRE trial has been published elsewhere. ${ }^{8}$ Briefly, VAMPIRE is a randomised trial on the value of blood-test ordering for patients with unexplained complaints. The study had two main objectives combined in one trial. The first main objective was to determine the accuracy of diagnostic blood tests for patients presenting with unexplained complaints, both when tests are ordered immediately and when they are postponed. The second main objective was to evaluate the effects of a systematically designed quality-improvement strategy for GPs, aiming at the postponement of blood-test ordering. Details on these objectives, on primary and secondary outcomes, as well as on power calculation can be found in the published protocol article. ${ }^{8}$ To serve these two main objectives, participating general practices were randomised to three groups. Group 1 was instructed to order blood tests immediately, and groups 2 and 3 to postpone

\section{How this fits in}

Unexplained fatigue is frequently encountered in general practice, and blood

tests are often ordered immediately for these patients, whereas initial

postponement of blood-test ordering for 4 weeks may be a sound alternative.

From this study it emerges that, in their decision making, there is a need for

GPs to balance up the yield of immediate, expanded test ordering with the high

risk of false-positive test results on one hand, and the diagnostic delay in the

relatively few diagnoses when postponing blood-test ordering on the other,

considering that the majority of patients do not revisit their GP for their

complaints within 4 weeks.

testing for 4 weeks. GPs in group 3 were supported by a systematically developed quality-improvement strategy.

To achieve allocation concealment, study groups were assigned to GPs by a random number seed computer program that randomised in blocks and was operated by an experienced research assistant. In this report, one of the pre-specified analyses is addressed. A distinction was therefore made between the immediate test ordering group (group 1) and the postponement group (groups 2 and 3 together). It was decided to randomise at practice level to prevent contamination between patients and individual GPs.

Initially, regional laboratories in the southern and western parts of the Netherlands were asked to participate in the trial. Subsequently, the GPs using the facilities of these laboratories were asked to participate and to include each consecutive eligible patient from February 2002 to December 2003. Patients were eligible for inclusion if they were 18 years of age or older and presented new unexplained complaints. Complaints were defined as new if they had not been presented to the GP within the previous 6 months. The complaint groups defined were fatigue, abdominal complaints, musculoskeletal complaints, weight changes, or itching. Their complaint had to be 'unexplained' in accordance with the definition given in the Dutch College of General Practitioners' guideline on blood-test ordering: those complaints for which a GP, after clarifying the reason for the encounter, taking the patient's history, and performing a physical examination, is unable to establish a diagnosis. Only patients who were able to read, speak, and understand Dutch were included. Patients with unexplained complaints were excluded if their GPs experienced a sense of alarm, making watchful waiting unacceptable. GPs provided their patients with written information and asked them for their informed consent. Patients were instructed to revisit their GPs if their complaints had not eased after 4 weeks. 


\section{Data collection}

For each patient, GPs filled in a case record form after the first consultation and again if a patient revisited the practice on account of the same complaint. The case record form contained questions regarding: the patient's history (for example, type of complaint, duration of complaints before presentation, symptoms); physical examination; and consultation as a whole (the degree to which the GP considered the complaint to be unexplained, the degree of certainty about the absence of sense of alarm, the working hypothesis, and the GP's satisfaction).

Depending on their allocation, GPs ordered blood tests immediately upon the first consultation or postponed blood-test ordering. When blood tests were ordered, either at the first consultation or when a patient re-consulted after 4 weeks, two prespecified sets of tests were performed: a limited set of tests as advised by the Dutch College of General Practitioners (haemoglobin, erythrocyte sedimentation rate, glucose, and thyroid-stimulating hormone tests), and an expanded complaint-specific set of tests. The tests in the latter set had been selected by an expert panel of GPs and clinical specialists $(n=20)$. Each panel member was asked

\section{Box 1. Complaint group-specific test set for fatigue (limited test set ${ }^{\mathrm{a}}$ and 13 extra tests).}

- Alkaline phosphatase

- Alanine aminotransferase

- Aspartate aminotransferase

- Carbohydrate-deficient transferrin

- Creatinin

Differentiated leukocyte count

- Erythrocyte sedimentation rate

Ferritin

- Gamma-glutamyl transferase

- Glucose

Haemoglobin

- Potassium

Lactate dehydrogenase

Leukocyte count

- Monosticon

- Thyroid-stimulating hormone

- Transferrin saturation

${ }^{a}$ Tests in italics are part of the limited Dutch College of General Practitioners' test set. to propose tests that they regarded to be useful in the work-up of patients with one out of five unexplained complaints in general practice. The five complaint groups were unexplained fatigue, abdominal complaints, musculoskeletal complaints, weight change, and itch. To prevent the comparison of separate test sets and diagnoses linked to different complaint groups, this paper is restricted to the presentation of the fatigue-specific set and thus to patients with unexplained fatigue. For the fatiguespecific set, all tests mentioned by two or more of the experts ( $n=17$; the limited set and 13 extra tests) were included (Box 1). The regional laboratories' reference values were used to determine test result norms. A test set result was called 'abnormal' if one or more test results were outside the reference range.

Electronic medical records were searched for complaint-related GP entries (for example, diagnoses, working hypotheses, and complaint-level evaluations) during a 1-year follow-up period. A final categorisation of complaints was performed in a three-step process. First, two of the authors independently searched the electronic medical records for all complaint-related GP entries and summarised these into one diagnosis per patient. Secondly, these patient-specific summaries were categorised into the following groups:

- Somatic illnesses detectable by blood-test ordering (for example, diabetes mellitus, hypothyroidism);

- Somatic illnesses not detectable by blood-test ordering (for example, fatigue due to viral upper respiratory tract infection, post-viral infection fatigue);

- Psychosocial illnesses (for example, depression, burn-out);

- Syndromes (for example, irritable bowel syndrome, chronic fatigue syndrome);

- Complaints not otherwise specified (for example, fatigue, tiredness); or

- Other (for all evaluations in the electronic medical records stated with a question mark, or listings of differential diagnoses).

In steps 1 and 2, consensus was reached by discussion. In case of disagreement, two members of the research team - both experienced GPs arbitrated. Finally, the categories were dichotomised as 'somatic illnesses detectable by blood-test ordering', or otherwise.

\section{Statistical analysis}

Two-by-two tables were constructed by combining results of the blood-test ordering (test set result 
abnormal: yes/no) and somatic illness detectable by blood-test ordering (yes/no). Two-by-two tables were also constructed for the limited test set and the fatigue-specific test set, and for the immediate test ordering group and the group tested after postponement.

To determine independent predictors for abnormal test results, a multivariate logistic regression model was used. The dependent variable was an abnormal test set (yes/no); the nine candidate predictors were the patient's sex; age; reported duration of complaint before presentation ( $\geq 4$ or $<4$ weeks); presence of psychosocial factors (yes/no/unknown); presence of previous episodes (>6 months ago) of unexplained fatigue (yes/no); abnormal findings on physical examination (yes/no); the GP's subjective assessment of the degree to which this episode of fatigue was unexplained (0 [totally unexplained] to 6 [slightly unexplained]); the GP's subjective assessment of the degree of certainty about the absence of a sense of alarm (0 [totally uncertain] to 6 [totally certain]); and the GP's certainty about the working hypothesis (0 [totally uncertain] to 6 [totally certain]).

Determinants with more than two categories were modelled as dummy variables. Analyses were performed using SPSS (version 12.02) and STATA software (version 10).

\section{RESULTS}

\section{General characteristics}

A total of 91 GPs were randomised, nine of whom withdrew before patient inclusion actually started, either for personal reasons or because of a lack of time. Of the remaining 82 GPs, 19 failed to include any patients. So, 63 GPs, affiliated with nine laboratories, included 513 patients with unexplained complaints (fatigue, abdominal complaints, musculoskeletal complaints, weight changes, or itching). This paper concerns only patients suffering from unexplained fatigue, of whom there were 325 . Background characteristics of GPs and patients are presented in Appendix 1.

Data from the electronic medical records of 29 patients are missing because they withdrew from the study or left the general practice. Data on blood-test ordering and follow-up from 296 patients, included by $60 \mathrm{GPs}$, were available for analysis (Figure 1).

\section{Immediate test ordering}

The 26 GPs who were randomised to order blood tests immediately included 158 patients. Blood tests were ordered immediately in 146 patients. Twenty-seven of the 138 patients whose GPs had been randomised to postpone blood-test ordering nevertheless underwent blood-test ordering at the first consultation. Thus, blood tests were ordered immediately for 173 patients.
Figure 1. Total numbers of patients in randomised (sub)groups and actual re-consultation and bloodtest ordering figures.

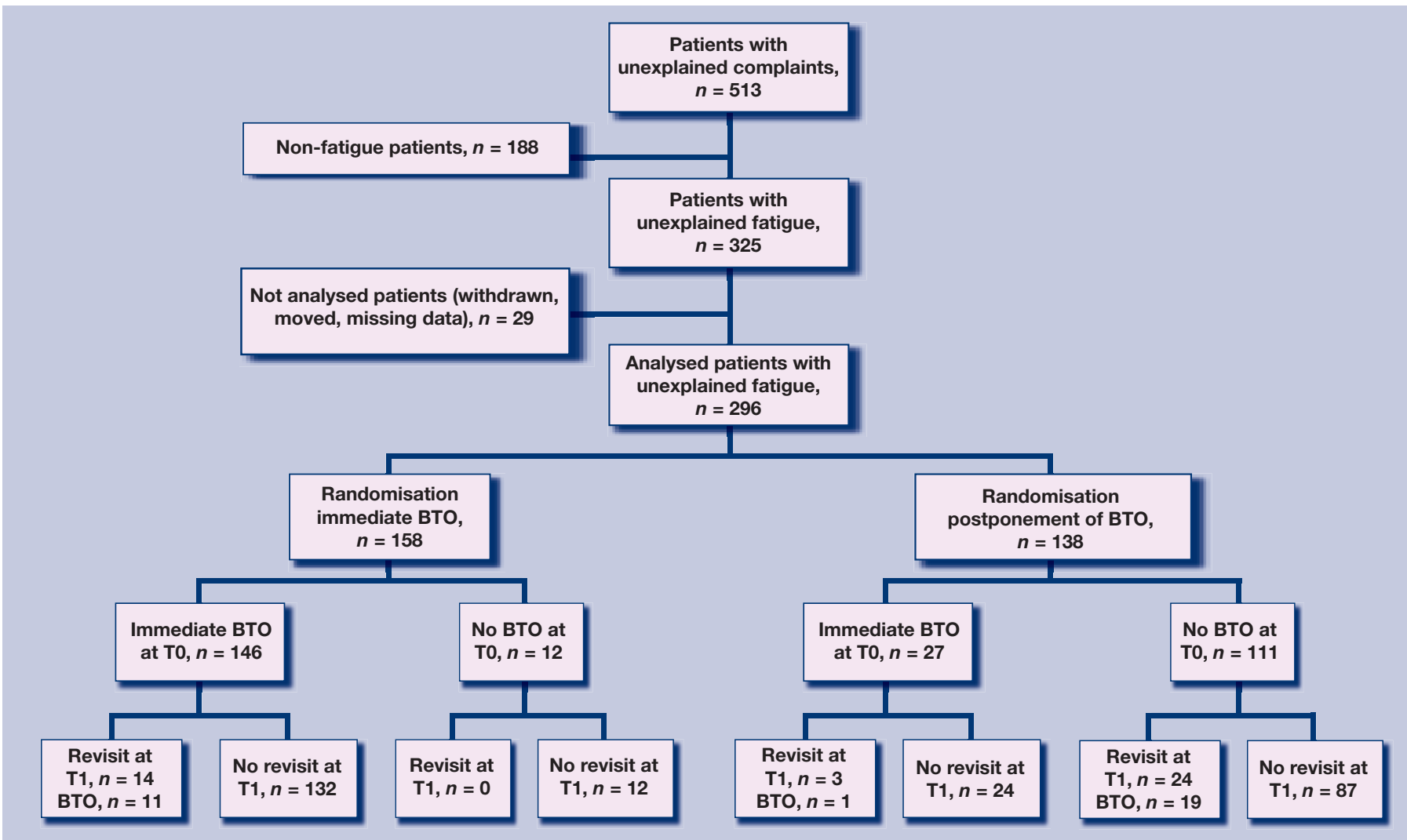

$\mathrm{BTO}=$ blood-test ordering. $\mathrm{TO}=$ consultation at presentation of complaints. $\mathrm{T} 1=$ consultation 4 weeks after presentation of complaints. 
Table 1a-d. Two-by-two tables relating test results to diagnoses in all tested patients.

Table 1a: limited (DCGP) test set T0

\begin{tabular}{lccc}
\hline Limited test set T0 & $\begin{array}{c}\text { Somatic } \\
\text { disease }^{\mathrm{a}}\end{array}$ & $\begin{array}{c}\text { No somatic } \\
\text { disease }\end{array}$ & Totals \\
\hline Abnormal test results & A: 11 & B: 38 & 49 \\
\hline Normal test results & C: 3 & D: 121 & 124 \\
\hline Totals & 14 & 159 & 173 \\
\hline
\end{tabular}

Table 1c: limited (DCGP) test set T1

\begin{tabular}{lccc}
\hline Limited test set T1 & $\begin{array}{c}\text { Somatic } \\
\text { disease }^{\text {a }}\end{array}$ & $\begin{array}{c}\text { No somatic } \\
\text { disease }\end{array}$ & Totals \\
\hline Abnormal test results & A: 1 & B: 6 & 7 \\
\hline Normal test results & C: 1 & D: 11 & 12 \\
\hline Totals & 2 & 17 & 19
\end{tabular}

Table 1b: expanded (fatigue-specific) test set T0

\begin{tabular}{lccc}
\hline Expanded test set T0 & $\begin{array}{c}\text { Somatic } \\
\text { disease }^{\mathrm{a}}\end{array}$ & $\begin{array}{c}\text { No somatic } \\
\text { disease }\end{array}$ & Totals \\
\hline Abnormal test results & A: 13 & B: 96 & 109 \\
\hline Normal test results & C: 1 & D: 63 & 64 \\
\hline Totals & 14 & 159 & 173 \\
\hline
\end{tabular}

Table 1d: expanded (fatigue-specific) test set T1

\begin{tabular}{lccc} 
Expanded test set T1 & $\begin{array}{c}\text { Somatic } \\
\text { disease }\end{array}$ & $\begin{array}{c}\text { No somatic } \\
\text { disease }\end{array}$ & Totals \\
\hline Abnormal test results & A: 2 & B: 11 & 13 \\
\hline Normal test results & C: 0 & D: 6 & 6 \\
\hline Totals & 2 & 17 & 19 \\
\hline
\end{tabular}

Somatic disease that may be detected by means of blood tests. DCGP = Dutch College of General Practitioners.

$T O=$ consultation at presentation. $T 1=$ second consultation 4 weeks after presentation.

In Table 1a (limited set) and Table 1b (fatiguespecific set), the two-by-two tables of all these immediately tested patients are presented. Fourteen out of 173 patients (8\%) turned out to have a somatic illness that could be detected by blood-test ordering. These illnesses are listed in Table 2.

Patients in cell $\mathrm{A}$ of Table $1 \mathrm{a}$ and Table $1 \mathrm{~b}$ (true positives) had a somatic illness that could be detected by blood-test ordering, and also showed abnormal test results in either the limited set $(n=11$, Table 1a) or the fatigue-specific set $(n=13$, Table 1b). The two extra patients who were diagnosed by the fatigue-specific set turned out to have infectious mononucleosis.

Patients in cell B (false positives) showed abnormal test results in the limited set $(n=38$, Table 1a) or in the fatigue-specific set ( $n=96$, Table 1b). Obviously, for reasons that were not analysed, these abnormal test results did not result in a diagnosis of a somatic illness detectable by blood-test ordering that related to that specific abnormal test result. They were therefore considered false-positive test results. For the limitedset, erythrocyte sedimentation rate and glucose, and for the fatigue-specific set, ferritin, gamma-glutamyl transferase, and carbohydrate-deficient transferrin were most often considered false positive.

Cell $C$ represents patients whose test results were not abnormal, although they were diagnosed with disorders that could have been detected by bloodtest ordering (false negatives; limited set $n=3$, two patients with infectious mononucleosis and one with dust mite allergy [patient that was considered to be tired because of long-lasting rhinitis complaints], Table 1a; fatigue-specific set $n=1$, the patient with dust mite allergy, Table 1b).

Cell D shows the 'true negatives': patients who had neither a somatic illness that could be detected by blood-test ordering, nor any abnormal test results (limited set $n=121$, Table 1a; fatigue-specific set $n=$ 63, Table 1b).

The striking difference between the limited and fatigue-specific sets of tests is the numbers of patients in cell $B$, the false positives (38 versus 96 , respectively).

Twenty-seven out of 138 patients from the postponement group had their blood tests ordered immediately. Obviously, GPs did not manage to postpone the blood-test ordering in these patients. From the two-by-two tables of this group of patients (data not shown), it became clear that, compared to the immediate test ordering group, a similar percentage of patients had a somatic illness that could be detected by blood-test ordering (2/27 [7.4\%] versus 14/173 [8\%]). The difference between the limited set and the fatigue-specific set in the number of patients in cell $B$ is also in line with the findings from the immediate test ordering group ( $7 / 27$ versus $13 / 27)$. The two patients with somatic illnesses that could be detected by blood-test ordering in this group turned

Table 2. Established diagnoses detectable with blood-test ordering ( $n=14)$.

\begin{tabular}{lc} 
Diagnosis & $n$ \\
\hline Diabetes mellitus & 4 \\
\hline Anaemia & 3 \\
\hline Infectious mononucleosis & 3 \\
\hline Hypothyroidism & 1 \\
\hline Dust mite allergy & 1 \\
\hline Hemoglobin E thalassemia & 1 \\
\hline Vitamin B12 deficiency & 1 \\
\hline Total & 14 \\
\hline
\end{tabular}

British Journal of General Practice, April 2009 
out to have type 2 diabetes mellitus and infectious mononucleosis respectively. In the Netherlands, full blood count is a separate test order from haemoglobin. Therefore, the infectious mononucleosis was not suspected on the basis of the results of the limited set alone, and was only discovered by the fatigue-specific set which also contained differentiated leukocyte count and the Monosticon test.

\section{Postponement of test ordering}

Of the 138 patients who were randomised to postponed blood-test ordering, 111 did not have their blood tests ordered immediately. Twenty-four of them re-consulted their GPs after 4 weeks, and 19 of these 24 had their blood tests ordered for the first time at that second consultation. In Table 1c and d, two-by-two tables for the limited set and the fatiguespecific set are presented. The one patient in cell $A$ of Table 1c (true positive, limited set) turned out to have diabetes mellitus. The one patient in cell $C$ of Table 1c (false negative, limited set) turned out to have haemochromatosis, with abnormal test results in the fatigue-specific set pointing towards this (abnormal ferritin and transferrin saturation test results). Therefore, in Table 1d, this patient moved to cell A (true positive, fatigue-specific set). If this had been approached as an 'intention to treat' or, in this case, an 'intention to postpone' analysis, the relative number of false-positive results would have been even lower, namely 6/111 for the limited test set and $11 / 111$ for the fatigue-specific one. However, it is not known what test results patients who did not reconsult, and thus were not tested, would have had.

The distribution of patients over the four cells of the two-by-two tables is comparable to the distribution in the immediate test ordering tables, although the numbers are too small to draw firm conclusions.

\section{Independent predictors}

It was not possible to define consultation-related independent indicators of abnormal test results; in other words, no determinants were retained in the logistic regression model. The multivariate odds ratios and their 95\% confidence intervals are given in Appendix 2 for both the limited set and the fatiguespecific set.

\section{DISCUSSION}

\section{Summary of main findings}

This study evaluated the recommendations of the Dutch College of General Practitioners' guideline on blood-test ordering for patients with unexplained complaints in general practice. This evaluation concerned both the recommended limited test set and the moment of blood-test ordering. In addition, the study explored whether there were consultationrelated independent determinants of abnormal test results. These results might help in careful consideration of the decision to order blood tests.

Eight per cent of patients turned out to have a somatic illness that could be detected by blood-test ordering. The expanded fatigue-specific set was found to have a minimal influence on the number of true positives (11/14 versus 13/14). One test only (for infectious mononucleosis) was responsible for this small increase in diagnostic yield. In contrast, the number of false-positive test results increased dramatically with the number of tests ordered. In this patient group in particular, where prior probabilities of somatic pathology are low, false-positive test results are undesired. False-positive test results may be harmful to patients. Some argue that they may result in cascades of unnecessary repeated bloodtest ordering, additional diagnostic procedures, redundant medication, or even referral to secondary care, hospitalisation, and surgery. ${ }^{9,10}$ Unfavourable additional effects of such cascades are patient anxiety, somatisation, high costs, iatrogenic somatic fixation, and the development of chronic illness behaviour. ${ }^{11,12}$ The question is whether this cascade actually takes place in patients with unexplained complaints in general practice. It may well be that GPs slightly stretch reference values when confronted with an unexpected borderline abnormal test result, and do not always start the cascade. ${ }^{13}$

Due to the small number of patients re-consulting their GPs after 4 weeks because of unresolved complaints, and because of the incorporation bias, little can be said about the effects of the postponement of blood-test ordering on the diagnostic value. However, the distribution of patients over the cells in the two-by-two tables in the postponement group did not differ remarkably from the immediate blood-test ordering group. The kinds of somatic illnesses that were detected by blood-test ordering were also similar. Although GPs may feel differently about diagnostic delay, the authors feel that their findings do not undermine the advice to postpone blood-test ordering for medical reasons.

No independent consultation-related determinants of abnormal test results were found in the set of variables studied, and, consequently, it is not possible to define which factors should influence GPs' decisions regarding blood-test ordering in patients with unexplained fatigue.

\section{Strengths and limitations of the study}

Most of the diagnoses from the category 'somatic illness detectable by blood-test ordering' were actually partly established by abnormal test results (for example, anaemia by low haemoglobin, diabetes 
by high glucose, hypothyroidism by high thyroidstimulating hormone). This incorporation bias unfortunately precluded the valid estimation of sensitivity, specificity, or predictive values of bloodtest ordering, because it would cause biased overestimation of these parameters.

For the presentation of the data, the data of all patients who had their blood tests ordered at the first consultation were combined, regardless of the randomisation group of their GPs. This influenced the results of the two-by-two tables at baseline. Twoby-two tables were constructed for the 27 patients from the postponement group, whom nevertheless had their blood tests ordered at baseline as well, to find out whether there were striking differences. This was not the case. Furthermore, the kind of diagnoses established in this group was not different.

\section{Comparison with existing literature}

The authors are not aware of other literature concerning the role of blood-test ordering for patients with unexplained complaints in general practice. Earlier research from the present group has already shown that watchful waiting in patients with unexplained complaints is feasible, does not cause additional anxiety or dissatisfaction in patients,,$^{14}$ and is cheaper than immediate test ordering.

\section{Implications for clinical practice}

These findings, in combination with (a) the low reconsultation rate of patients after 4 weeks, (b) the lack of striking differences in diagnoses and blood test results in the postponement group compared to the immediate test ordering group, and (c) the finding that the limited set is as good as or even better than the expanded fatigue-specific one (with less risk of false positives), reinforce the advice of the Dutch College of
General Practitioners: try to postpone blood-test ordering in patients with initially unexplained fatigue, and if blood tests are ordered, limit the tests to haemoglobin, erythrocyte sedimentation rate, glucose, and thyroid-stimulating hormone. GPs, in their decisions to order blood tests immediately or try to postpone this, need to balance up the yield of immediate, elaborate test ordering with the high risk of false-positive test results on one hand, and the diagnostic delay in the relatively few diagnoses when postponing blood-test ordering on the other, considering that the majority of patients do not revisit their GP for their complaints within 4 weeks.

\section{Funding body}

Dutch College of Health Care Insurances (CVZ); Netherlands Organization of Scientific Research (NWO)

\section{Ethical approval}

The medical ethics review boards of both the Academic Medical Center-University of Amsterdam and the University Medical Centre Maastricht have approved the study

\section{Competing interests}

The authors have stated that there are none

\section{Acknowledgements}

We would like to acknowledge all patients for participation and all GPs for their help in patient recruitment in this study.

\section{Discuss this article}

Contribute and read comments about this article on the Discussion Forum: http://www.rcgp.org.uk/bjgp-discuss

\section{REFERENCES}

1. Dinant GJ, Wijk van MAM, Janssens HJEM, et al. Dutch College of General Practitioners guideline 'Blood testing. General principles and use by GP' [NHG-standaard Bloedonderzoek. Algemene principes en uitvoering in eigen beheer; In Dutch]. Huisarts Wet 1994; 37: 202-211.

2. Koch $\mathrm{H}$, van Bokhoven MA, ter RG, et al. Demographic characteristics and quality of life of patients with unexplained complaints: a descriptive study in general practice. Qual Life Res 2007; 16(9): 1483-1489.

3. Kenter EG, Okkes IM, Oskam SK, Lamberts H. Tiredness in Dutch family practice. Data on patients complaining of and/or diagnosed with 'tiredness'. Fam Pract 2003; 20(4): 434-440.

4. Dekay ML, Asch DA. Is the defensive use of diagnostic tests good for patients, or bad? Med Decis Making 1998; 18(1): 19-28.

5. Owens DK. Defensive diagnostic testing - a case of stolen utility? Med Decis Making 1998; 18(1): 33-34.

6. Rovner DR. Laboratory testing may not glitter like gold. Med Decis Making 1998; 18(1): 32-33.

7. Hindmarsh JT, Lyon AW. Strategies to promote rational clinical chemistry test utilization. Clin Biochem 1996; 29(4): 291-299.

8. Van Bokhoven MA, Koch H, van der WT, et al. Blood test ordering for unexplained complaints in general practice: the VAMPIRE randomised clinical trial protocol. [ISRCTN55755886]. BMC Fam Pract 2006; 7: 20.

9. Fink P. Surgery and medical treatment in persistent somatizing patients. J Psychosom Res 1992; 36(5): 439-447.

10. Fink $P$. The use of hospitalizations by persistent somatizing patients. Psychol Med 1992; 22(1): 173-180.

11. Sherwood P, Lyburn I, Brown S, Ryder S. How are abnormal results for liver function tests dealt with in primary care? Audit of yield and impact. BMJ 2001; 322(7281):276-278.

12. Vafiadis P. The dilemma of the unexpected result. Aust Fam Physician 1996; 25(6): 971-973.

13. Houben PHH, van der Weijden T, van Bokhoven MA, et al. Overwegingen van huisartsen bij het interpreteren van laboratoriumonderzoek; een kwalitatief onderzoek [In Dutch]. Huisarts Wet 2005; 48(7): 326-332.

14. Van Bokhoven MA, Koch H, van der Weijden T, et al. Influence of watchful waiting on satisfaction and anxiety among patients seeking care for unexplained complaints. Ann Fam Med 2009; 7: 112-120. 


\section{Appendix 1. Background data of participating GPs and included patients.}

\begin{tabular}{|c|c|c|c|}
\hline \multirow[b]{2}{*}{ GPs } & \multirow[b]{2}{*}{$\begin{array}{c}\text { All GPs, } \\
n=63\end{array}$} & \multicolumn{2}{|c|}{ Randomisation } \\
\hline & & $\begin{array}{c}\text { Group 1: } \\
\text { immediate BTO, } n=27\end{array}$ & $\begin{array}{c}\text { Group 2: } \\
\text { postponement of BTO, } n=36\end{array}$ \\
\hline Age, mean years (SD) & $46(6.7)$ & $47.4(4.6)$ & $45(7.9)$ \\
\hline \multicolumn{4}{|l|}{ Sex, $n(\%)$} \\
\hline Male & $47(74.6)$ & $21(77.8)$ & $26(72.2)$ \\
\hline Female & $16(25.4)$ & $6(22)$ & $10(27.8)$ \\
\hline Experience, mean number of years (SD) & $14(8.4)$ & $14.1(6.4)$ & $13(9.6)$ \\
\hline \multicolumn{4}{|l|}{ Organisation of practice, $n(\%)$} \\
\hline Soloists & $22(35)$ & $8(29.6)$ & $14(38.9)$ \\
\hline Other & $41(65)$ & $19(70.4)$ & 22 (61.6) \\
\hline \multicolumn{4}{|l|}{ Practice location, $n(\%)$} \\
\hline Urban & $33(52.4)$ & $13(48.1)$ & $20(55.6)$ \\
\hline Semi-rural & $30(47.6)$ & $14(51.9)$ & $16(44.4)$ \\
\hline Patients & $\begin{array}{c}\text { All patients, } \\
n=325\end{array}$ & $\begin{array}{c}\text { Group 1: } \\
\text { immediate BTO, } n=166\end{array}$ & $\begin{array}{c}\text { Group 2: } \\
\text { postponement of BTO, } n=159\end{array}$ \\
\hline Age, mean years (SD) & $41.4(15.7)$ & $41.2(15)$ & $41.6(16.5)$ \\
\hline \multicolumn{4}{|l|}{ Sex, $n(\%)$} \\
\hline Male & $93(28.6)$ & $46(27.7)$ & $47(29.6)$ \\
\hline Female & $232(71.4)$ & $120(72.3)$ & $112(70.4)$ \\
\hline \multicolumn{4}{|l|}{ Type of health insurance, $n(\%)$} \\
\hline Private & $101(31.1)$ & $55(33.1)$ & $46(28.9)$ \\
\hline Public & $194(59.7)$ & $101(60.8)$ & $93(58.5)$ \\
\hline \multicolumn{4}{|l|}{ Highest level of education, $n(\%)$} \\
\hline Elementary & $21(6.5)$ & $10(6.0)$ & $11(6.9)$ \\
\hline Secondary & $161(49.6)$ & $87(52.4)$ & $74(46.6)$ \\
\hline Higher & $114(35)$ & $60(35.5)$ & $54(38.8)$ \\
\hline
\end{tabular}

BTO $=$ blood-test ordering .

\section{Appendix 2. Multivariable logistic regression.}

\begin{tabular}{|c|c|c|c|}
\hline Variables & Categories & OR limited set ${ }^{a}(95 \% \mathrm{Cl})$ & $\begin{array}{l}\text { OR expanded (fatigue- } \\
\text { specific) test set }(95 \% \mathrm{Cl})\end{array}$ \\
\hline Patient's sex & Female & $0.6(0.3$ to 1.3$)$ & $0.9(0.4$ to 2$)$ \\
\hline Patient's age & Years & 1 (0.99 to 1.04$)$ & $1(0.98$ to 1$)$ \\
\hline Duration of complaints & More than 4 weeks & 0.8 (0.3 to 2.2$)$ & 0.9 (0.3 to 2.3$)$ \\
\hline Presence of psychosocial factors & $\begin{array}{c}\text { Yes } \\
\text { Unknown } \\
\text { No }\end{array}$ & $\begin{array}{c}1 \text { (reference category) } \\
0.7(0.3 \text { to } 2.2) \\
0.6(0.2 \text { to } 1.5)\end{array}$ & $\begin{array}{c}1 \text { (reference category) } \\
1.8(0.6 \text { to } 5.6) \\
0.6(0.3 \text { to } 1.3)\end{array}$ \\
\hline Previous episodes of unexplained fatigue & Yes & $1(0.4$ to 2.4$)$ & $0.8(0.4$ to 1.8$)$ \\
\hline Abnormal physical examination & Yes & $2.1(0.7$ to 6.2$)$ & $2(0.6$ to 6.2$)$ \\
\hline Degree to which complaint is unexplained according to GP & $0-6$ scale & 0.9 (0.8 to 1.1$)$ & $0.9(0.7$ to 1.1$)$ \\
\hline GP's certainty about absence of sense of alarm & $0-6$ scale & $1.2(0.8$ to 1.7$)$ & $1.3(0.9$ to 1.9$)$ \\
\hline GP's certainty about working hypothesis & $0-6$ scale & $1(0.7$ to 1.5$)$ & 0.8 (0.6 to 1.1$)$ \\
\hline
\end{tabular}

aDutch College of General Practitioners' test set. OR = odds ratio. 